\title{
Evaluation of Quality of Life in Patients Treated with Traditional Chinese Medicine
}

\author{
Serkan Sertel ${ }^{*}, 1,2,4$, Henry Johannes Greten ${ }^{4,5}$, Hans-Joachim Kraemer ${ }^{4}$, Thomas Efferth ${ }^{2,3}$, \\ Peter K. Plinkert ${ }^{1}$ and Ingo Baumann ${ }^{1}$
}

\author{
${ }^{1}$ Department of Otorhinolaryngology, Head \& Neck Surgery, University of Heidelberg, Im Neuenheimer Feld 400, \\ D-69120 Heidelberg, Germany \\ ${ }^{2}$ German Cancer Research Centre (DKFZ), Pharmaceutical Biology (C015), Im Neuenheimer Feld 280, D-69120 \\ Heidelberg, Germany \\ ${ }^{3}$ Department of Pharmaceutical Biology, Institute of Pharmacy and Biochemistry, University of Mainz, Staudinger Weg \\ 5, D-55099 Mainz, Germany \\ ${ }^{4}$ German Society of Traditional Chinese Medicine, Karlsruherstr. 12, D-69126 Heidelberg, Germany \\ ${ }_{5}^{5}$ Instituto de Ciencias Biomedicas Abel Salazar (ICBAS), University of Porto, Largo Professor Abel Salazar 2, 4099-003 \\ Porto, Portugal
}

\begin{abstract}
The worldwide clinical implementation of Traditional Chinese Medicine (TCM) is increasing as complementary therapy to Western Medicine. This development leads to an increasing demand to evaluate not only the scientific proof of efficacy, but also to measure the quality of life (QOL) concepts specific to TCM.

This article attempts to 1) review the relevant published literature (indexed in Medline) on the evaluation of QOL in patients treated with TCM, 2) compare QOL of patients treated with Western Medicine and TCM and 3) describe the limitations of the literature and future research directions.

Overall, the approach of TCM as a model of system biology appears to improve QOL and may be worth integrated into conventional Western Medicine.
\end{abstract}

Keywords: Traditional Chinese Medicine (TCM), Quality of Life (QOL), complementary therapy, western medicine.

\section{TRADITIONAL CHINESE MEDICINE (TCM)}

\subsection{Definition}

A modern understanding of Chinese Medicine (CM) regards TCM as a model of system biology with the objective of a holistic therapy of diseases. Diagnosis in TCM is based on a system of clinical signs, sensations and findings designed to assess the functional vegetative state of the patient. Therapy includes vegetative and central nervous reflex interventions such as acupuncture and Chinese manual therapy (Tuina), traditional vegetative biofeedback exercises (Taichi and Qigong), which consist of meditative breathing and movement exercises. The main therapeutic method, however, is Chinese phytopharmacology and dietetics, which includes administration of plants $[1,2]$, minerals, and other products, as well as Chinese functional food.

\subsection{History}

Although the actual origin of $\mathrm{CM}$ remains unclear, it seems that CM has a history of more than 5000 years [3].

*Address correspondence to this author at the Department of Otorhinolaryngology, Head \& Neck Surgery, University of Heidelberg, Im Neuenheimer Feld 400, D-69120 Heidelberg, Germany; Tel: +49 6221/56 39502; Fax: +49 6221/56 7478;

E-mail: serkan.sertel@med.uni-heidelberg.de
The oldest written sources are the I Ging (Book of Changes) and the Huangdi Neijing (Yellow Emperor's Classic on Internal Medicine) dating back over 2300 years. The first contains mathematical knowledge of binary numbers [4] (yang and yin as $\mathrm{I} / 0$ ) which was decoded by the mathematician Gottfried Wilhelm Leibniz (1646-1716), the latter is comparable in importance to the Hippocratic Corpus in Greek medicine [5].

The term "Traditional Chinese Medicine" (TCM) originally describes the modern practice of $\mathrm{CM}$ as a result of widespread reforms that took place after 1950 in the People's Republic of China. This included the need of quick distribution of the methodology, resulting in a certain reduction of the theoretical background.

The term "Classical Chinese medicine" (CCM) often refers to medical practices that are based on theories and methods dating from before the fall of the Qing Dynasty (1911) [6].

"Integrated Chinese Medicine" or integrated TCM refers to more comprehensive recompilations based on CCM and the current status quo of methodologies. The theoretical basis of such recompilations, e.g. the "Heidelberg Model of $T C M$ " [2, 4] are mathematical models on vegetative regulation inherent in the classical corpus medicus. By this approach, certain technical terms like yin, yang and the 
phases can be translated as vegetative functional terms. This may considered crucial for the integration of TCM in Western health care systems and research.

\subsection{Distribution}

Western Medicine, like any other corpus medicus, has limitations in diagnostic and therapeutic success [7]. This is why 60 to $80 \%$ of the chronically ill search for complementary treatment methods such as acupuncture [813]. In fact, according to the World Health Organization, in many developed countries, $70-80 \%$ of the population has used some form of alternative or complementary medicine (e.g., acupuncture) [14]. The report from a Consensus Development Conference on Acupuncture held at the National Institutes of Health in 1997 stated that acupuncture is being "widely" practiced-by thousands of physicians, dentists, and other practitioners-for relief or prevention of pain and for various other health conditions. According to the 2002 National Health Interview Survey-the largest and most comprehensive survey of complementary and alternative medicine use by American adults to date-an estimated 8.2 million U.S. adults had ever used acupuncture, and an estimated 2.1 million U.S. adults had used acupuncture in the previous year [15]. In the year 2003, Chinese Medicine, including its products and services, had an estimated turnover of 3,2 billion $€$, which is roughly $40 €$ per citizen of Germany per year [2].

\subsection{Clinical Studies on Acupuncture in Otorhinolaryngo- $\log \mathbf{y}$}

Diseases in Otorhinolaryngology offer a wide range of possible application of TCM. In one of our first studies, we could show that additional use of acupuncture on top of nonsteroidal anti-inflammatory drugs effectively reduced posttonsillectomy pain [16]. Nevertheless, major challenges in clinical acupuncture studies remain the design of (I) double blinding and (II) appropriate placebo controls [17].

Double blinding is the scientific gold standard in pharmaceutical studies. As acupuncture is a complex procedure, it is difficult to design studies in which both the acupuncturist and patient are blinded to the treatment.

Recently, we could meet these standardized, scientific requirements in a controlled, double-blinded acupuncture study with the "Heidelberg double-blinding array". We confirmed a significant decongestant efficacy of a specific verum acupuncture to a non-specific control acupuncture and a non-acupuncture standard medication group for the improvement of nasal breathing [18].

\subsection{TCM and Phytopharmacology}

Medicinal herbs have played an important role in Western medicine from ancient to modern times. However, medicinal plants gradually lost their importance as pharmaceutical synthetic chemistry progressed in Western countries during the 20 th century. Currently, there is a revival of interest in medicinal plants and an increasing scientific interest in phytochemicals as chemical lead compounds for the generation of semi-synthetic derivatives [19-21].

Hundreds of botanical, animal, and mineral preparations were categorized in traditional Chinese pharmacopoeias starting millennia ago. TCM plants provide an enormous variety of drugs based on thousands of years of tradition [22, 23]. Consequently, it can be assumed that by this time many ineffective prescriptions have disappeared from the system of TCM thereby significantly improving the prospects for identifying novel active constituents from TCM-plant prescriptions [24]. Among anticancer agents, camptothecin, paclitaxel, vincristine, and indirubin are developed from Camptotheca acuminata, Taxus chinensis, Catharanthus roseus, and Baphicacanthus cusia, respectively, although the original plants were not used traditionally to treat cancer [20].

\section{QUALITY OF LIFE (QOL)}

\subsection{Definition}

The World Health Organization (WHO) defines quality of life (QOL) as the individual perception of the position in life within the context of the culture and value systems and in relation to individual goals, expectations, standards and concerns [25].

QOL can be differentiated in the assessment of general and health-related QOL, which both are regarded as a multidimensional construct [26]. According to Lawton et al. [27], four main aspects belong to QOL: objective environment, competence of behavior (including health), perceived QOL and psychological well-being (including life satisfaction) [27].

\subsection{Measures of QOL}

Among the various possibilities to assess QOL, two questionnaires have been applied in the majority of QOLassessments in patients treated with TCM.

The World Health Organization Quality of Life Survey (WHOQOL-100) is a standard QOL assessment questionnaire consisting of 100 questions. By this test, the overall QOL and the general health perceptions are assessed. The WHOQOL was developed especially to be a crosscultural measurement tool. It has demonstrated excellent reliability and validity [28, 29]. Chinese [30], Danish [31], and Portuguese [32] translations have subsequently been tested and confirmed good reliability and validity.

The SF-36 is a multi-purpose, short-form health survey with 36 questions. It consists of a profile of functional health and wellbeing scores, as well as general physical and mental health self-assessments. Accordingly, the SF-36 has shown to be useful in surveys of the general population and specific subpopulations, thereby comparing the relative burden of diseases in order to evaluate the health benefits offered by a wide range of different treatments [33]. The SF-36 has been translated for use in more than 40 countries $[34,35]$.

\section{INTRODUCTION OF QOL ASSESSMENT IN TCM}

As TCM is a medical system with proper diagnostic key symptoms and signs, the development of health-related Quality of life (HRQOL) assessments specific to TCM was mandatory. For this purpose, the Chinese Quality of Life Instrument (ChQOL) was developed, which is a TCMspecific self-report health status instrument. The ChQOL is a 50 item-questionnaire, which comprises aspects of health, which are not well covered by the existing generic health 
related QOL scores, such as the WHOQOL-100 and the SF36. The ChQOL categorizes physical aspects of health in two domains, the physical form domain and the mental domain. Moreover, it contains a domain on emotion that covers various kinds of moods. This domain gathers information, which belongs to the affective self-perception in nature. Other psychological mechanisms, for example, self-esteem, that are more cognitive are however not included [36].

Speaking of the limitations, there is less overlapping in the WHOQOL-100 and the ChQOL at the domain level. There is even less overlapping between SF-36 and ChQOL. However, the combination of ChQOL and WHOQOL-100 or SF-36 is claimed to provide a wider range of information on people's health. Due to methodological limitations, the structure of the ChQOL was not able to fully reflect the concept of health in TCM. As an example, although the pulse diagnosis is one of the principle findings in TCM, it could not be included in the CHQOL [36].

The content of ChQOL was shown to be valid in the context of TCM for Cantonese speaking Chinese independent of spoken dialect and health care system [37].

In the USA, a structured assessment instrument was developed for use in clinical trials of acupuncture and other Chinese medical therapies. It is using an interdisciplinary approach. Under the guidance of two group process facilitators, and in order to establish whether the assessment instrument was consistent with accepted CM diagnostic categories (face validity) and included the full range of each concept's meaning (content validity), a panel of TCM expert clinicians convened and their responses were organized using the Delphi process, an iterative, anonymous, ideagenerating and consensus-building process. An aggregate rating measure was obtained by taking the mean of mean ratings for each question across all 10 experts. The assessment instrument, called TEAMSI-TCM (Traditional East Asian Medicine Structured Interview, TCM version) uses the pattern differentiation model characteristic of TCM. This modular, dynamic version was specifically designed to assess women, with a focus on gynecologic conditions; with modifications it can be adapted for use with other populations and conditions. TEAMSI-TCM is a prescriptive instrument that guides clinicians to use the proper indicators, combines them in a systematic manner, and generates conclusions. In conjunction with treatment and training it may serve to increase inter-rater reliability and inter-trial reproducibility in CM clinical trial [38].

\section{EVALUATION OF QOL IN TCM}

\subsection{Otorhinolaryngology \& Pulmonology}

An acupuncture-like transcutaneous nerve stimulation method (Codetron) without invasive needles was developed to mimic acupuncture treatment. In a Phase I-II study, the effectiveness of Codetron in treating radiation-induced xerostomia in head-and-neck cancer patients was examined. Xerostomia symptoms were assessed by a five-item xerostomia symptom questionnaire with a Visual Analogue Scale and QOL was evaluated using the Head and Neck Radiotherapy Questionnaire (QOL-RTI) [39]. A significant improvement in whole saliva production and related symptoms was noted at 3 and 6 months after treatment completion, respectively. However, no statistically significant change in the QOL evaluation compared with baseline was observed [40].

An Austrian group of investigators assessed lung function and QOL by the Pediatric Asthma Quality of Life Questionnaire [41] in asthmatic school-age children. In a randomized, placebo-controlled, double-blinded pilot study, patients were treated with laser acupuncture and oral probiotic drops (living non-pathogenic Enterococcus faecalis). Patients of the control group were treated with a laser pen, which did not emit laser light and were given placebo drops. No additional beneficial effects of TCM treatment was seen on forced expiratory volume in $1 \mathrm{~s}$ (FEV1), QOL and use of rescue medication in children with intermittent or mild persistent bronchial asthma [42].

Neumeister et al. [43] investigated the effect of acupuncture in patients with chronic obstructive pulmonary disease (COPD) on QOL with the Quality-of-life for Respiratory Illness Questionnaire (QOL-RIQ) [44], pulmonary function measures and inspiratory mouth occlusion pressures (MOP) in a prospective randomized and placebo-controlled pilot study. Patients receiving verum acupuncture improved significantly with FEV1, residual volume (RV) and total lung capacity (TLC). There was an improvement of large magnitude in QOL and a trend of lower demand of the respiratory pump. In the placebo group, only a slight improvement of QOL, a deterioration of lung function parameters and a trend of higher demand of the respiratory pump were observed.

\subsection{Oncology}

The emotional distress of a diagnosis of cancer and the persistent side effects while having treatment significantly compromise patients' QOL [45]. As a consequence, those diagnosed with cancer are increasingly seeking out supportive and complementary therapies as adjuncts to medical treatment in their efforts to cope with their illness and promote healing. Cancer patients are frequent users of complementary medicine, $91 \%$ of cancer patients diagnosed with cancer in the United States [46] use some form of complementary medicine when receiving usual medical treatment. While some patients hope these will impact on survival, many are using these methods to improve their QOL.

Over the last three decades, there has been a growing consensus among health care providers and researchers that efficacy of therapeutic intervention should be evaluated by its impact on both quantity and quality of life [47]. In oncology or palliative care, QOL assessment is even more important in determining treatment benefit when survival advantage is limited and other aspects of the patients' life are more important [48]. It may also provide valuable clinical data to support treatment decisions for the patient and doctor to decide on the best treatment options.

Mistletoe extracts have been applied to cancer patients for several decades. The influence of a standardized mistletoe extract (sME) on QOL in breast, ovarian and nonsmall cell lung cancer patients was investigated in a prospective randomized controlled clinical study. QOL improved significantly for patients who were complementarily treated with sME, as determined by the 
questionnaires FLIC (Functional Living Index-Cancer), Traditional Chinese Medicine Index and the KPI (Karnofsky Performance Index) in comparison to the control group [49].

The European Organization for Research and Treatment of Cancer (EORTC) developed cancer-specific core questionnaires, one common to patients with various sites of primary tumor, and also site-specific questionnaires for the measurement of QOL of patients with cancers of specific sites of the body. The EORTC QLQ-C30 was proved to have good reliability and validity [3-8]. The EORTC QLQ-C30 has been translated into traditional Chinese (Mandarin spoken in Taiwan) and validated in Taiwan [9-12]. The EORTC QLQ-OV28 was designed as a supplement to the EORTC QLQ-C30 for the use in ovarian cancer clinical trials and related studies [13] and has been used in a study assessing QOL for patients after pelvic exenteration [14]. Cultural differences between Taiwan and China seem not to influence EORTC QLQ-OV28. The reliability and validity of the Taiwan Chinese Version of the EORTC QLQ-OV28 on patients with ovarian cancer in Taiwan was confirmed [50].

QOL of cancer patients is often diminished due to the side effects of treatment and symptoms of the disease itself. TCM provides Qigong, a Chinese meditative practice in combination with slow gentle body movements and controlled breathing techniques, is supposed to promote the circulation of $q i$ within the human body, and enhance a practitioner's overall health [1]. Oh et al. [51], indicate that Qigong with usual medical treatment is able to improve QOL (measured with the EORTC QLQ-C 30) of cancer patients and reduce inflammation (measured with the creactive protein) in cancer patients. However, the results were not statistically significant between treatment and control groups, most probably due to small sample size.

The success of cancer chemo- and radiotherapy is mainly measured in terms of disease-free survival, whereas QOL is often separated from survival. This may be inadequate, and the full potential of beneficial treatment effects should be taken into account. Hence, health-related quality of life (HRQL) represents an outcome, which also has to be incorporated in randomized, controlled clinical trials. This has been considered not only as relevant from the patient's perspective gaining cure from the disease, but also from a health policy level. The old "Who should pay?" dilemma is even more pressing in economically critical times, where decisions have to be made, how much a society is able to pay for expensive therapies [52].

Survival analyses, e.g., Kaplan-Meier statistics, measure two health states: dead or alive. Such mortality-based statistics do not consider morbidity of surviving cancer patients. The overall survival time of cancer patients can be divided into time with toxicity, time without symptoms and toxicity (TWiST), and time after systemic relapse (REL) [53]. Various health outcome models have been proposed focusing on QOL of cancer patients, such as quality of wellbeing (QWB), quality-adjusted life years (QALY), or health years equivalent (HYE) [54].

While these models may be generally considered in medicine, the Q-TWiST method has been proposed for use in cancer trials. Q-TWiST describes the quality-adjusted time without symptoms and toxicity. It integrates side effects and benefits of adjuvant cancer therapy by determination of mortality, morbidity, and duration of different health states in a single number [55-60].

Q-TWiST is a well-established method in clinical oncology. Its value for TCM has, however, not been elucidated yet. From our point of view, the performance of Q-TWiST-based clinical trials integrating TCM in cancer therapy is one of the most thriving and attractive challenges in therapy research today. As recently reviewed [61], there is mounting evidence that TCM reduces side effects associated with cancer chemo- and radiotherapy. Therefore, measuring Q-TWiST using TCM would greatly foster the integration of TCM in standard cancer treatment regimens.

In this context, two general TCM categories may be discussed, which are suitable for setting up Q-TWiST-based clinical trials:

1. Phytotherapy: A recent meta-analysis showed that Astragalus-based herbal remedies improved response to chemotherapy and reduced therapy-related side effects by stimulation of macrophages and natural killer cells as well as inhibition of T-helper cell type 2 cytokines [62]. A total of 34 randomized clinical trials ( $n=2815$ patients $)$ with platinum-based chemotherapy for non-small cell lung cancer were included in the meta-analysis. In 12 trials $(n=940$ patients), a reduced risk of death at 12 months appeared. Better response to chemotherapy was found in 30 trials ( $n=2472$ patients). Injection of the Chinese $A i D i$ remedy improved life quality in four trials ( $n=257$ patients) as measured by Karnovsky index.

2. Acupuncture is widely used in TCM for pain treatment, a major side effect of cancer therapy, e.g. in Otorhinolaryngology. The value of acupuncture was re-evaluated in a meta-analysis, which included 11 randomized controlled trials, two non-randomized trials and 8 case studies [63]. The authors found that acupuncture, electro-acupuncture, and massage therapy indeed significantly relieved pain of cancer patients. Typical side effects of cancer chemotherapy are nausea and vomiting. As described in another meta-analysis considering 11 clinical trials $(n=1247$ patients), acupuncture-point stimulation, electroacupuncture, and acupressure reduced the incidence of acute vomiting, but not acute or delayed nausea severity as compared to control [64].

\section{CONCLUSIONS}

Screening the literature, indexed in Medline, few relevant studies have adequately addressed the evaluation of QOL in patients treated with TCM. Reports in the literature have been conflicting, with some studies finding deterioration in QOL and some finding stability or improvement in QOL over time.

Given the fact that QOL reflects patients' perceptions of their health state, QOL is an especially pertinent issue on which to focus. Before concluding anything regarding QOL two premises are, however, important to note. First, it is important to utilize a well-validated measure of QOL in order to compare QOL in patients treated with TCM. 
Second, due to the relatively small number of studies concerning this issue, any interpretation of the impact of TCM on QOL must be tentative.

Finally, recommendations are difficult to make based on the research. There have been no prospective studies in which patients have been randomly assigned to Western treatment or TCM treatment only. Despite the limitations of the current research, we can draw a tentative conclusion. In sum, the approach of TCM as a system biology appears to improve QOL and may be worth integrated into conventional Western Medicine.

\section{PERSPECTIVES}

Future research concerning the evaluation of QOL in patients treated with TCM should include prospective and multi-center studies, which would allow comparative analysis of QOL. Also, studies measuring the impact of various TCM treatment methods on QOL should be undertaken, as there appear to be adverse reactions from traditional herbal medicine.

Ideally, these studies would assist Western physicians as well as patients in the process of decision making whether to implement TCM in the Western treatment modalities as an add on treatment to conventional cancer therapy.

\section{REFERENCES}

[1] Porkert M. The Theoretical Foundations of Chinese Medicine MIT Press; 1974.

[2] Greten HJ. Kursbuch Traditionelle Chinesische Medizin. 2. ed. Stuttgart - New York: Thieme Publishing House 2006.

[3] Liu Y. The Essential Book of Traditional Chinese Medicine: Theory, Clinical Practice: Columbia University Press 1988.

[4] Greten HJ. Checkliste Chinesische Phytotherapie. 1. ed. Stuttgart New York: Hippokrates Publishing House 2009.

[5] Sivin N. Huang ti nei ching. Loewe M, Ed. Berkley and Los Angeles: University of California Press 1993.

[6] http: //en.wikipedia.org/wiki/TraditionalV̈ChineseV̉medicine. [09.10.09].

[7] Kroenke K, Mangelsdorff AD. Common symptoms in ambulatory care: incidence, evaluation, therapy, and outcome. Am J Med 1989; 86(3): 262-6.

[8] Ernst E. Acupuncture--how effective is it really? Fortschr Med 1998 20; 116(1-2): 20-6.

[9] Ernst E. The risks of complementary therapy methods. Fortschr Med 1998 20;116(1-2): 28-30.

[10] White AR, Ernst E. A trial method for assessing the adequacy of acupuncture treatments. Altern Ther Health Med 1998; 4(6): 66-71.

[11] Richardson J. The use of randomized control trials in complementary therapies: exploring the issues. J Adv Nurs 2000; 32(2): 398-406.

[12] Bernstein BJ, Grasso T. Prevalence of complementary and alternative medicine use in cancer patients. Oncology (Williston Park) 2001; 15(10): 1267-72; discussion 72-8, 83.

[13] Ashikaga T, Bosompra K, O'Brien P, Nelson L. Use of complimentary and alternative medicine by breast cancer patients: prevalence, patterns and communication with physicians. Support Care Cancer 2002; 10(7): 542-8.

[14] WHO. www.who.int/mediacentre/factsheets/fs134/ en/. [08.10.09].

[15] NIH. www.nccam.nih.gov/health/acupuncture/ introduction.htm. [08.10.09].

[16] Sertel S, Herrmann S, Greten HJ, et al. Additional use of acupuncture to NSAID effectively reduces post-tonsillectomy pain. Eur Arch Otorhinolaryngol 2009; 266(6): 919-25.

[17] White AR, Filshie J, Cummings TM. Clinical trials of acupuncture: consensus recommendations for optimal treatment, sham controls and blinding. Complement Ther Med 2001; 9(4): 237-45.

[18] Sertel S, Bergmann Z, Ratzlaff K, Baumann I, Greten HJ, Plinkert PK. Acupuncture for nasal congestion: A prospective, randomized, double-blind, placebo-controlled clinical pilot study. Am J Rhinol Allergy 200918.

[19] Efferth T, Li PC, Konkimalla VS, Kaina B. From traditional Chinese medicine to rational cancer therapy. Trends Mol Med 2007; 13(8): 353-61.

[20] Efferth T, Fu YJ, Zu YG, Schwarz G, Konkimalla VS, Wink M. Molecular target-guided tumor therapy with natural products derived from traditional Chinese medicine. Curr Med Chem 2007; 14(19): 2024-32.

[21] Efferth $T$, Miyachi $H$, Bartsch $H$. Pharmacogenomics of a traditional Japanese herbal medicine (Kampo) for cancer therapy. Cancer Genomics Proteomics 2007; 4(2): 81-91.

[22] Tang W, Hemm I, Bertram B. Recent development of antitumor agents from chinese herbal medicines; part I. Low molecular compounds. Planta Med 2003; 69(2): 97-108.

[23] Tang W, Hemm I, Bertram B. Recent development of antitumor agents from Chinese herbal medicines. Part II. High molecular compounds(3). Planta Med 2003; 69(3): 193-201.

[24] Newman DJ, Cragg GM. Natural products as sources of new drugs over the last 25 years. J Nat Prod 2007; 70(3): 461-77.

[25] http: //www.who.int/mentalV̉health/whoV̈qolV̇fieldV்trialV்1995. WHOQOL. 1995 [08.10.09].

[26] Browne JP, O'Boyle CA, McGee HM, et al. Individual quality of life in the healthy elderly. Qual Life Res 1994; 3(4): 235-44.

[27] Lawton MP, Moss M, Glicksman A. The quality of the last year of life of older persons. Milbank Q 1990; 68(1): 1-28.

[28] Development of the World Health Organization WHOQOL-BREF quality of life assessment. The WHOQOL Group. Psychol Med 1998; 28(3): 551-8.

[29] Power M, Harper A, Bullinger M. The World Health Organization WHOQOL-100: tests of the universality of Quality of Life in 15 different cultural groups worldwide. Health Psychol 1999; 18(5): 495-505.

[30] Wang GHH, Zang D. Quality of life of nurses in a general hospital. Chin Ment Health J 2001; 15(5): 308-11.

[31] Norholm V, Bech P. The WHO Quality of Life (WHOQOL) Questionnaire: Danish validation study. Nord J Psychiatry 2001; 55(4): 229-35.

[32] Fleck MP, Chachamovich E, Trentini C. Development and validation of the Portuguese version of the WHOQOL-OLD module. Rev Saude Publica 2006; 40(5): 785-91.

[33] Ware JE, Jr., Sherbourne CD. The MOS 36-item short-form health survey (SF-36). I. Conceptual framework and item selection. Med Care 1992; 30(6): 473-83.

[34] Ware JE, Jr, Kosinski M, Gandek B, et al. The factor structure of the SF-36 Health Survey in 10 countries: results from the IQOLA Project. International Quality of Life Assessment. J Clin Epidemiol 1998; 51(11): 1159-65.

[35] Alonso J, Ferrer M, Gandek B, et al. Health-related quality of life associated with chronic conditions in eight countries: results from the International Quality of Life Assessment (IQOLA) Project. Qual Life Res 2004; 13(2): 283-98.

[36] Leung KF, Liu FB, Zhao L, Fang JQ, Chan K, Lin LZ, Development and validation of the Chinese Quality of Life Instrument. Health Qual Life Outcomes 2005; 3: 26.

[37] Wong W, Lam CL, Leung KF, Zhao L. Is the content of the Chinese Quality of Life Instrument (ChQOL) really valid in the context of traditional Chinese medicine in Hong Kong? Complement Ther Med 2009; 17(1): 29-36.

[38] Schnyer RN, Conboy LA, Jacobson E, et al. Development of a Chinese medicine assessment measure: an interdisciplinary approach using the delphi method. J Altern Complement Med 2005; 11(6): 1005-13.

[39] Trotti A, Johnson DJ, Gwede C, et al. Development of a head and neck companion module for the quality of life-radiation therapy instrument (QOL-RTI). Int J Radiat Oncol Biol Phys 1998 1; 42(2): 257-61.

[40] Wong RK, Jones GW, Sagar SM, Babjak AF, Whelan T. A Phase I-II study in the use of acupuncture-like transcutaneous nerve stimulation in the treatment of radiation-induced xerostomia in head-and-neck cancer patients treated with radical radiotherapy. Int J Radiat Oncol Biol Phys 2003 1; 57(2): 472-80.

[41] Juniper EF, Guyatt GH, Feeny DH, Ferrie PJ, Griffith LE, Townsend M. Measuring quality of life in children with asthma. Qual Life Res 1996; 5(1): 35-46. 
[42] Stockert K, Schneider B, Porenta G, Rath R, Nissel H, Eichler I. Laser acupuncture and probiotics in school age children with asthma: a randomized, placebo-controlled pilot study of therapy guided by principles of Traditional Chinese Medicine. Pediatr Allergy Immunol 2007; 18(2): 160-6.

[43] Neumeister W, Kuhlemann H, Bauer T, Krause S, SchultzeWerninghaus G, Rasche K. Effect of acupuncture on quality of life, mouth occlusion pressures and lung function in COPD. Med Klin (Munich) 1999; 94(1 Spec No): 106-9.

[44] Maille AR, Koning CJ, Zwinderman AH, Willems LN, Dijkman $\mathrm{JH}$, Kaptein AA. The development of the 'Quality-of-life for Respiratory Illness Questionnaire (QOL-RIQ)': a disease-specific quality-of-life questionnaire for patients with mild to moderate chronic non-specific lung disease. Respir Med 1997; 91(5): 297309.

[45] Pearman T. Quality of life and psychosocial adjustment in gynecologic cancer survivors. Health Qual Life Outcomes 2003; 1: 33.

[46] Cassileth BR, Deng G. Complementary and alternative therapies for cancer. Oncologist 2004; 9(1): 80-9.

[47] Dancey J, Zee B, Osoba D, et al. Quality of life scores: an independent prognostic variable in a general population of cancer patients receiving chemotherapy. The National Cancer Institute of Canada Clinical Trials Group. Qual Life Res 1997; 6(2): 151-8.

[48] Efficace F, Bottomley A. Assessing HRQOL: a neglected issue for high-grade glioma. Lancet Oncol 2003; 4(1): 11-2.

[49] Piao BK, Wang YX, Xie GR, et al. Impact of complementary mistletoe extract treatment on quality of life in breast, ovarian and non-small cell lung cancer patients. A prospective randomized controlled clinical trial. Anticancer Res 2004; 24(1): 303-9.

[50] Chie WC, Lan CY, Chiang C, Chen CA. Quality of life of patients with ovarian cancer in Taiwan: validation and application of the Taiwan Chinese version of the EORTC QLQ-OV28. Psychooncology 2009; Sept 23 [Epub ahead of print].

[51] Oh B, Butow P, Mullan B, Clarke S. Medical Qigong for cancer patients: pilot study of impact on quality of life, side effects of treatment and inflammation. Am J Chin Med 2008; 36(3): 459-72.

[52] Kaplan RM. Quality of life assessment for cost/utility studies in cancer. Cancer Treat Rev 1993; 19(Suppl A): 85-96.
[53] Goldhirsch A, Gelber RD, Simes RJ, Glasziou P, Coates AS. Costs and benefits of adjuvant therapy in breast cancer: a quality-adjusted survival analysis. J Clin Oncol 1989 ; 7(1): 36-44

[54] Rusthoven JJ. Are quality of life, patient preferences, and costs realistic outcomes for clinical trials? Support Care Cancer 1997; 5(2): 112-7.

[55] Feldstein ML. Quality-of-life-adjusted survival for comparing cancer treatments. A commentary on TWiST and Q-TWiST. Cancer 1991 1; 67(3 Suppl): 851-4.

[56] Gelber RD, Goldhirsch A, Cavalli F. Quality-of-life-adjusted evaluation of adjuvant therapies for operable breast cancer. The International Breast Cancer Study Group. Ann Intern Med 1991 15; 114(8): 621-8.

[57] Gelber RD, Goldhirsch A, Cole BF. Evaluation of effectiveness: QTWiST. The International Breast Cancer Study Group. Cancer Treat Rev 1993; 19 Suppl A: 73-84.

[58] Gelber RD, Goldhirsch A, Cole BF, Wieand HS, Schroeder G, Krook JE. A quality-adjusted time without symptoms or toxicity (Q-TWiST) analysis of adjuvant radiation therapy and chemotherapy for resectable rectal cancer. J Natl Cancer Inst 1996 7; 88(15): 1039-45.

[59] Stephens R. Clinically important differences in Q-TWIST - one twist too many? Qual Life Res 2006; 15(3): 425-6; discussion 7-8.

[60] Jang RW, Le Maitre A, Ding K, et al. Quality-adjusted time without symptoms or toxicity analysis of adjuvant chemotherapy in non-small-cell lung cancer: an analysis of the National Cance Institute of Canada Clinical Trials Group JBR.10 trial. J Clin Oncol 2009 10; 27(26): 4268-73.

[61] Konkimalla VB, Efferth T. Evidence-based Chinese medicine for cancer therapy. J Ethnopharmacol 2008 5; 116(2): 207-10.

[62] McCulloch M, See C, Shu XJ, et al. Astragalus-based Chinese herbs and platinum-based chemotherapy for advanced non-smallcell lung cancer: meta-analysis of randomized trials. J Clin Oncol 2006 20; 24(3): 419-30.

[63] Pan CX, Morrison RS, Ness J, Fugh-Berman A, Leipzig RM. Complementary and alternative medicine in the management of pain, dyspnea, and nausea and vomiting near the end of life. A systematic review. J Pain Symptom Manage 2000 ; 20(5): 374-87.

[64] Ezzo J, Vickers A, Richardson MA, et al. Acupuncture-point stimulation for chemotherapy-induced nausea and vomiting. J Clin Oncol 2005 1; 23(28): 7188-98. 